however, is far from continuous, showing breaks and concentrations as well as many branches or feathers which are well illustrated in the many photographs reproduced in the proceedings of this symposium. A major category of spiral galaxies has a ring structure in the central regions from which the spiral arms emerge; another group has a central bar which is the starting point for a double spiral arm pattern.

The Basel symposium brought together most of the optical, radio and theoretical astronomers who were working in the field of galactic and extragalactic structure. On the observational side the neutral hydrogen data give the best description of the spiral structure of our galaxy; two radically different interpretations of the observations have been made which have yet to be reconciled. As a consequence it is not at all clear how to join the various elongated features together in order to outline the possible continuous spiral structure. Optically, more data have become available in recent years and this has led to a good picture of the spiral structure near the Sun.

The renewed interest in the theoretical interpretations of spiral structure in galaxies was evident in the large number of papers presented on this topic at the symposium. The gravitational interpretation of the spiral structure seems now to be firmly established and has taken over completely from the magnetic field interpretation which had been popular in various forms for some 20 years. The general magnetic field is now known to have a strength of only 2-3 microgauss and is therefore unable to exert sufficient mechanical force to have any significant influence in producing spiral structure. It does, however, have an important role in influencing local galactic structure and, of course, in producing dust grain alignment, synchrotron radio emission and a number of other observable effects.

This book emphasizes the large amount of high quality observational material available, and demonstrates that a considerable amount of success has been achieved in explaining many of the observed phenomena.

The editors are to be commended for their speedy and pleasing production of this volume which will be valuable to workers in this and allied fields of $\begin{array}{ll}\text { astronomy. } & \text { R. D. DAviES }\end{array}$

\section{Life of Gauss}

Carl Friedrich Gauss: a Biography. By Tord Hall. Translated from the Swedish by Albert Froderberg. Pp. 176. (MIT: Cambridge, Massachusetts and London, December 1970.) £3.75.

THIs book is rather disappointing, for it adds little to our knowledge of Gauss. Tord Hall seems more concerned to instruct the reader in mathematics than to write a historical study of Gauss's work. His procedure is to indicate Gauss's ideas, say, on the geometry of curved surfaces, and then to provide illustrations of the mathematical techniques by means of examples. The primary purpose of this book is not a historical analysis of Gauss's mathematical achievements, and its success should therefore be judged with respect to the terms which the author has tried to satisfy. To me, the mathematical sections of this book are cogent, and are comprehensible to readers of modest mathematical attainment. The book contains an outline of Gauss's diverse fields of interest leavened by a pleasant biographical portrait, and serves as a useful introduction to a very difficult subject. Nevertheless, the sketch of Gauss's scientific personality in this book is far too anecdotal.

\section{P. M. Heimann}

\section{Homage to Richard Rado}

Studies in Pure Mathematics: Papers in Combinatorial Theory, Analysis, Geometry, Algebra, and the Theory of Numbers. Edited by L. M. Mirsky. Pp. viii +276. (Academic: London and New York, January 1971.) $£ 5.00$.

THE 26 papers in this volume are offered to Richard Rado in celebration of his sixty-fifth birthday. Such a birthday present is a tribute to the distinction of the mathematician and also to the abundant friendliness of the man. Rado was born in Berlin and studied at the universities of Berlin, Göttingen and Cambridge. After appointments in the mathematics departments of the University of Sheffield and King's College, London, he followed E. H. Neville in the chair of mathematics at the University of Reading in 1954 . Over 80 of his publications are listed in this book, and they range over a wide field of mathematics. His polished technique, reminiscent at times of the delicate stopvolley of a Wimbledon star, has helped him to clarify and to solve many problems; but his fundamental contributions to modern combinatorial theory, particularly in the establishment of the partition calculus, may perhaps be regarded as his most important and influential work.

The individual items deal with researches at a high level, connected as a rule with some aspect of Rado's own studies. One unusual paper is by van der Waerden; it describes an after-lunch session in which he, Artin and Schreier together arrive at a proof of Baudet's conjecture that, if the positive integers are divided into two classes, at least one of the classes will contain an arithmetic progression of arbitrary length. The literature of the psychology of mathe- matical invention is less plentiful than one would like ; mathematicians seldom have time or opportunity to explain how they made their discoveries, even if they can remember how tentative gropings and sudden illuminations eventually developed into rigorous demonstration. Hence van der Waerden's detailed reconstruction is a most interesting and valuable complement to the writings of Hadamard and Polya.

\section{T. A. A. Broadbent}

\section{Cut and Glue Topology}

Surgery on Compact Manifolds. By C. T. C. Wall. (London Mathematical Society Monographs, No. 1.) Pp. $x+$ 280. (Academic: London and New York, December 1970.) $£ 5.00$.

Geomerric topology has come a long way from the old party trick of cutting a Möbius band down the middle and producing a connected piece of paper. This technique of cutting up surfaces and glueing them together again has been generalized to higher dimensions and is now called surgery. Wall's book is an attempt to collate all the known results of surgery (plus a few more) and bind them all in one cover. So let me start by saying that this is a very important book and should be bought by anyone at all interested in topology.

The initial idea of surgery is reasonably easy to understand. One tries to cut up and reglue a space (or more correctly a continuous function between spaces) and get something simpler. The interest lies in the case where there is an obstruction to doing this. In the simply connected case, the obstructions vanish for odd dimensional spaces and for even dimensional spaces the signature and the Arf/Kervaire invariant are obstructions. In the non-simply connected case there are obstructions which lie in the Wall group of the fundamental groups. These groups are fearfully hard to pin down and have only been calculated in a few cases.

After some preliminary results and statements in chapters 0 and 1 , the author is already able by page 19 to find a counter-example to Mazur's relative non-stable neighbourhood theorem. In chapter 2, Poincaré complexes which are the algebraic analogue of a space are discussed. Then in chapter 3 there are some statements about the Wall groups with some proofs in chapter 4. In chapters 5 to 8 the book discusses the various special cases of even and odd dimensions, bounded and unbounded manifolds. In chapter 9 a proof of the principal theorem is given and some applications follow from chapter 10 onwards. These applications include manifold structures on Poincaré complexes, embeddings with submani- 